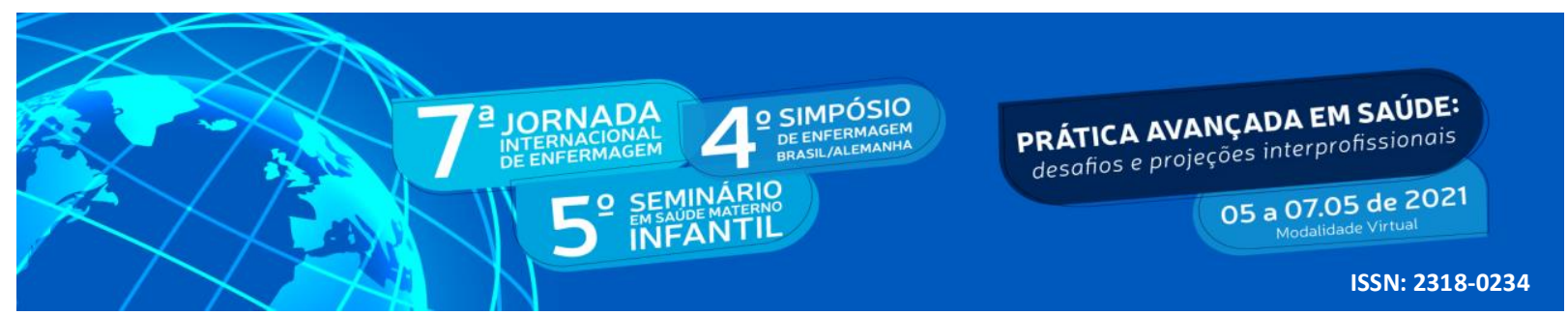

DOI: http://doi.org/10.48195/jie2021-083

\title{
PARTICIPAÇÃO PATERNA NO PARTO, NASCIMENTO E AMAMENTAÇÃO: RELATO DE EXPERIÊNCIA
}

\author{
Thanandra Freitas de Souza ${ }^{1}$; Leandro da Silva de Medeiros ${ }^{2}$; Carolina Fernandes da \\ Rocha $^{3}$; Gabriella Dalla Corte Córdova ${ }^{4}$; Janine Vasconcelos ${ }^{5}$
}

\begin{abstract}
RESUMO
Este estudo tem por objetivo descrever a experiência vivenciada por estudantes de graduação em enfermagem em relação a amamentação na primeira hora de vida e a participação paterna no parto e nascimento em uma maternidade de risco habitual. Trata-se de um estudo descritivo, do tipo relato de experiência, desenvolvido em uma instituição de ensino superior da região central do Rio Grande do Sul, no período de julho à agosto de 2020. Os resultados observados durante a vivência foram de suma importância para a figura paterna, tanto quanto para a criança e puérpera, tendo em vista que houve uma melhor fortificação do vínculo entre a família. Para nos estudantes foi satisfatório observar que essa relação proporciona que o parto seja um momento único, acolhedor e seguro à gestante. Conclui-se que a participação paterna em todos os momentos da gestação só traz benefícios positivos para a vida do progenitor, puérpera e bebê.
\end{abstract}

Palavras-chave: Aleitamento Materno; Enfermagem; Gestação; Participação Paterna.

\begin{abstract}
This study aims to describe an experience lived by undergraduate nursing students in relation to breastfeeding in the first hour of life and the paternal participation does not participate and birth in a usual risk maternity. This is a descriptive study, of the experience report type, developed in a higher education institution in the central region of Rio Grande do Sul, from July to August 2020. The results observed during the experience were extremely important for the paternal figure, as much as for a child and puerperal, considering that there was a better strengthening of the bond between the family. For students it was satisfactory to note that this relationship offers that childbirth is a unique, welcoming and safe moment for pregnant women. It is concluded that the paternal participation in all the moments of the gestation only brings positive benefits for the life of the parent, postpartum and baby.
\end{abstract}

Key Words: Breastfeeding; Nursing; Gestation; Fatherly participation.

\footnotetext{
1 Estudante do Curso de Enfermagem da Universidade Franciscana - UFN. E-mail: thanandrasouza03@gmail.com

${ }^{2}$ Estudante do Curso de Enfermagem da Universidade Franciscana - UFN. E-mail: leandro.medeiros@ufn.edu.br

${ }^{3}$ Estudante do Curso de Enfermagem da Universidade Franciscana - UFN. E-mail: carolinafernandessm@gmail.com

${ }^{4}$ Estudante do Curso de Enfermagem da Universidade Franciscana - UFN. E-mail: gabrielladcc@gmail.com

${ }_{5}^{5}$ Orientadora. Mestre em Saúde Materno Infantil. Docente do Curso de Enfermagem da Universidade Franciscana - UFN. E-mail: j.vasconcelos@ufn.edu.br
} 


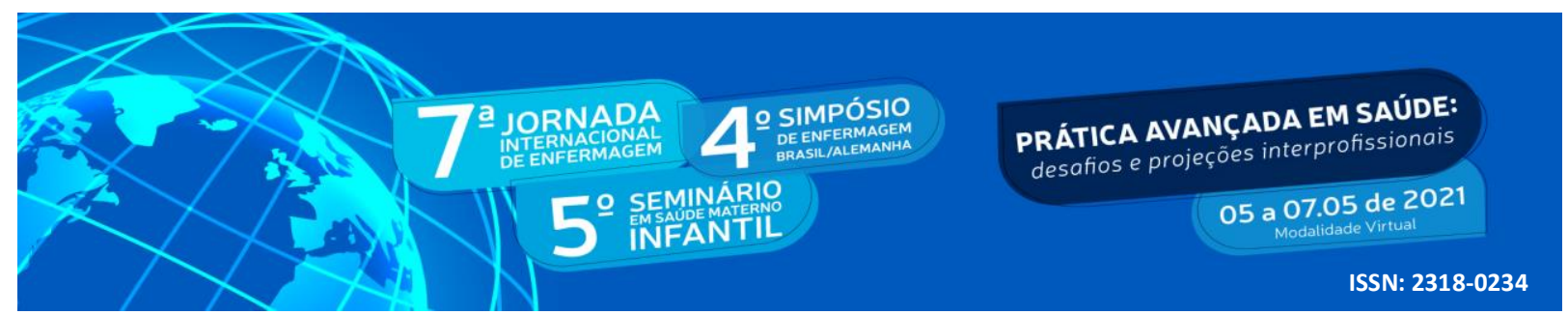

\section{INTRODUÇÃO}

A cada ano no Brasil ocorre cerca de três milhões de nascimentos, totalizando quase seis milhões de pessoas, na qual $98 \%$ destes nascimentos acontecem na rede hospitalar, sejam eles públicos ou privados (BRASIL, 2017). Isso significa que, a cada ano no Brasil, o nascimento influencia uma parcela significativa da população.

Dessa forma, o nascimento no ambiente hospitalar se caracteriza pela adoção de várias tecnologias e procedimentos com o objetivo de torná-lo mais seguro para a mulher. Se por um lado, o avanço da obstetrícia contribuiu com a melhoria dos indicadores de morbidade e mortalidade materna e perinatal, por outro, permitiu a concretização de um modelo que considera a gravidez, o parto e o nascimento como doenças e não como expressões de saúde (CÔRTES et al., 2018).

A assistência ao parto no Brasil, caracteriza-se pelo uso abusivo ou inadequado de intervenções e pelo cerceamento dos direitos da parturiente, tais como a amamentação na primeira hora de vida e restrições à presença do acompanhante em todos os períodos clínicos do parto (DONATO et al, 2018). Esses problemas influenciaram na elaboração de políticas públicas e na construção de protocolos de incentivo às boas práticas de atenção ao parto e nascimento.

As boas práticas de atenção ao parto e nascimento foram descritas primeiramente em 1996 pela Organização Mundial de Saúde (OMS) e atualizadas em 2018. Essas são práticas comuns determinadas para a condução do processo de parturição, baseadas em evidências científicas, com a finalidade de estabelecer cuidados adequados, seguros para a mulher e assegurar a qualidade da assistência materno-infantil (WHO, 2018).

Ressalta-se que, dentre as boas práticas, a amamentação na primeira hora de vida é considerada pela OMS uma medida que evita um milhão de mortes neonatais por ano, dentre estas, a participação paterna está relacionada as boas praticas trazendo mais segurança para a gestante. Nessa perspetiva, Sousa, et al (2020) diz que a participação paterna no parto traz maior segurança e felicidade da mulher, reduz o tempo do trabalho de parto, aumenta o vínculo familiar e diminui as intervenções medicamentosas durante o evento. 


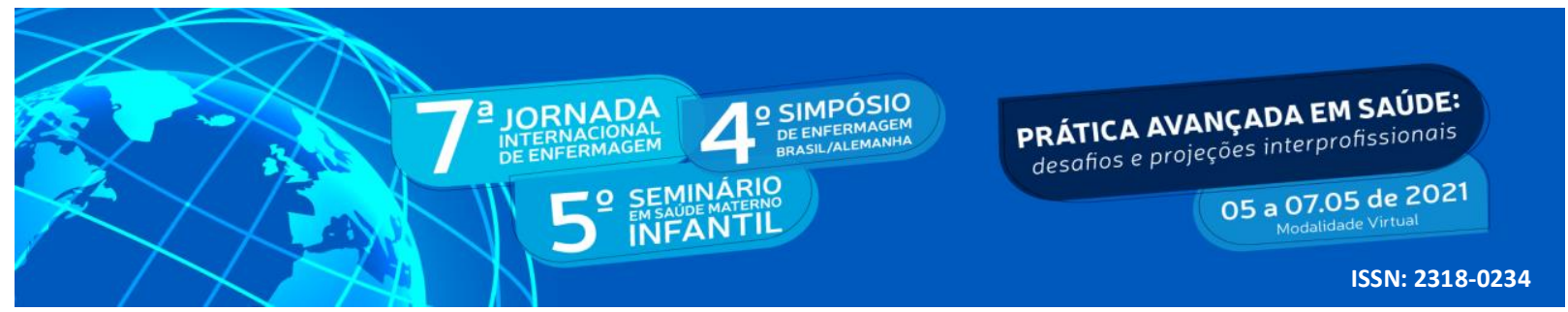

Dessa forma, este trabalho torna-se importante em virtude das consequências relacionadas a não adesão das boas praticas dentro do serviço de saúde, neste caso, a golden hour e a participação paterna dentre os diferentes momentos do parto. Assim, considerando a relevância da participação paterna no parto, nascimento e na amamentação, sobretudo, na primeira hora de vida, questiona-se: Quais as experiências de acadêmicos de enfermagem quanto à participação paterna no parto, no nascimento e na amamentação durante primeira hora de vida em uma maternidade de risco habitual?

\section{OBJETIVO}

Este estudo tem por objetivo descrever a experiência de acadêmicos de enfermagem quanto à participação paterna no parto, nascimento e amamentação na primeira hora de vida em uma maternidade de risco habitual.

\section{METODOLOGIA}

Trata-se de um estudo descritivo, do tipo relato de experiência. Segundo Ferreira (2019), este é um texto que descreve precisamente uma dada experiência que possa contribuir de forma relevante para sua área de atuação. Caracteriza-se pela descrição que um autor ou uma equipe fazem de uma vivência profissional tida como exitosa ou não, mas que contribua com a discussão, a troca e a proposição de ideias para a melhoria do cuidado na saúde.

$\mathrm{O}$ estudo foi realizado por 4 acadêmicos do $6^{\circ}$ e $8^{\circ}$ semestre do curso de Enfermagem de uma Instituição de Ensino Superior da região central do Rio Grande do Sul. Sendo desenvolvido na Maternidade de um hospital público de risco habitual no qual se caracteriza pelos seus atendimentos a demanda espontânea, o mesmo fica localizado na região central, a partir de atividades teórico-práticas propostas pela disciplina de "Atenção Integral à Saúde da Mulher" do Curso de graduação em Enfermagem, sob orientação da professora responsável.

A experiência em tela, aconteceu entre o período de julho a agosto de 2020, na maternidade, visto que este setor proporcionou aos acadêmicos de enfermagem realizarem o acompanhamento da gestante, desde sua chegada a maternidade até o seu momento de alta. Durante este processo, realizou-se um trabalho de orientação dos acadêmicos junto à figura 


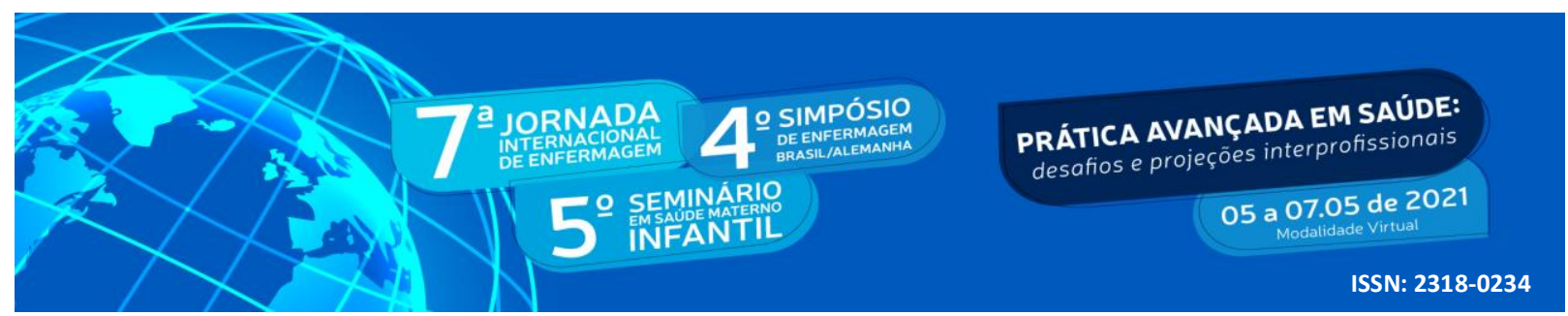

paterna. Os mesmos destacaram aos pais a sua importância durante todo o trabalho de parto, pós-parto e principalmente durante a amamentação, servindo de apoio psicológico e físico para a puérpera neste processo delicado.

\section{RESULTADOS E DISCUSSÃO}

Sabe-se que a amamentação na primeira hora de vida do recém-nascido (RN) é considerada como fator protetor para a mortalidade neonatal. Estudos científicos já comprovam este efeito protetor e redutor da mortalidade, devido à colonização intestinal de bactérias saprófitas encontradas no leite materno e aos fatores imunológicos bioativos adequados para o recém-nascido, presentes no colostro materno (ANTUNES et al, 2017).

Além disso, é de suma importância a participação ativa do progenitor em todos os momentos da gestação desde a consulta de pré-natal, trabalho de parto, parto, pós-parto e alta. Vale ressaltar que esses momentos em que a família está unida, fortalece ainda mais o vínculo entre os mesmos, além de trazer muitos benefícios para o pai, sejam estes afetivos e/ou emocionais.

É essencial destacar que quando o pai está por perto auxiliando e proporcionando apoio os índices de amamentação aumentam, uma vez que seu suporte neste processo, é fundamental para a mãe que alimenta a criança seja capaz de decidir entre a continuação ou não da amamentação, visto que esta nem sempre é uma tarefa fácil para as puérperas (BRASIL, 2020).

De maneira ampla, o tema paternidade e cuidado abrange o envolvimento ativo dos homens em todo o processo de gestação, parto, puerpério, amamentação -excepcionalmente- a Golden Hour, uma vez que oportuniza a criação de uma relação mais forte e saudável entre pais, mães e filhos(as) (BRASIL, 2016). Sabe-se que, a Golden Hour se faz presente na maioria dos partos que ocorrem na maternidade, só não é colocada em prática quando se tem necessidade de intervenção da equipe (em casos de risco à vida) da mãe e do recém-nascido.

Nesse sentido, sob o olhar dos acadêmicos de enfermagem, pôde-se analisar que a Golden Hour é incentivada pelos profissionais de saúde, uma vez que as puérperas, em sua grande maioria, aguardavam com expectativa e anseio por este momento. Inicialmente, notavase que as primeiras tentativas foram frustradas, visto que o recém-nascido apresentava dificuldade em fazer a pega correta, e por seguinte ouvia-se o choro do binômio no ambiente. 


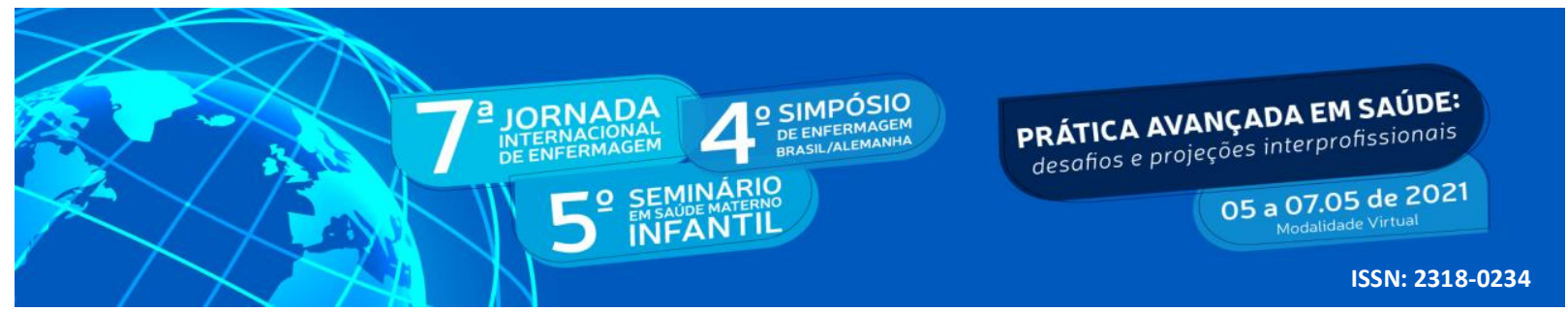

Dessa forma, construía-se uma comoção para auxiliar a puérpera e o seu bebê, de tal maneira que os acadêmicos auxiliaram no posicionamento correto para amamentação, à qual o tronco e o abdômen do bebê deve estar direcionado para a barriga da mãe. Assim, instruindo na pega correta, onde o queixo do recém-nascido deve encostar no seio, os lábios virados para o lado de fora e a boca deve sugar não somente o mamilo, mas sim grande parte da aréola (BRASIL, 2017).

Estas instruções possibilitaram que os anseios da mãe fossem amenizados em grande parte, no entanto, algumas continuavam apresentando dificuldades. Neste momento, notava-se que o pai não sabia como conduzir e auxiliar a puérpera e o bebê a fim de minimizar as barreiras encontradas no processo de amamentação. Vale ressaltar que as dificuldades encontradas pelo pai em prestar assistência, deve-se aos aspectos socioculturais marcados pela instituição do patriarcado, que provoca o distanciamento e obstáculos para o exercício da relação paternoinfantil (PINTO et al., 2018).

No entanto, é importante que a participação da figura paterna seja incentivada desde o pré-natal, uma vez que reforça os vínculos familiares e diminui o nível de ansiedade e depressão pós-parto da puérpera após a chegada do recém-nascido (BRASIL, 2012). Nesse aspecto, os acadêmicos estimulavam a participação paterna, e convidaram o mesmo para se incluir nas orientações voltadas para a amamentação e cuidados para com o recém-nascido.

No decorrer da vivência foi possível identificar que a maioria dos pais eram poucos engajados nesse processo, além de possuir muitas dúvidas, mas ficavam envergonhados e com receio de fazer questionamentos. Ao realizar uma atividade de educação em saúde interativa, acolhedora e confortável foi possível perceber através desta o vínculo e a promoção de uma assistência satisfatória e encantadora.

Além de oferecer informações adequadas para os pais e mães que se encontram permeados de dúvidas, medos e inseguranças em relação a essa nova fase é fundamental realizar uma escuta ativa e um diálogo com os mesmos. Atentando às necessidades, fragilidades e respeitando suas complexidades no contexto dos padrões culturais (crenças e valores), sociais e econômicos (OLIVEIRA et al., 2017). 


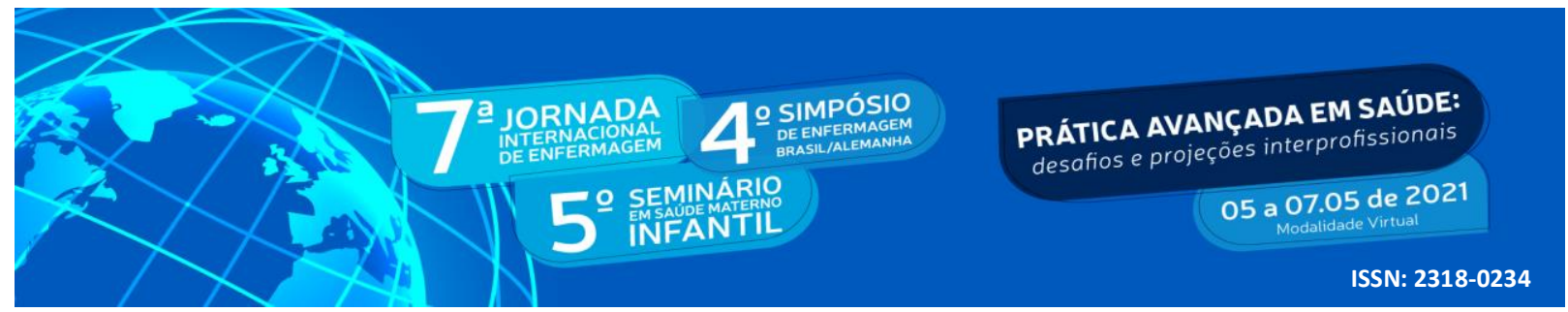

No entanto, para a enfermagem ainda há alguns fatores que dificultam a realização de boas condutas no pós-parto, como a sobrecarga de trabalho da equipe, bem como o desconhecimento das gestantes sobre os seus direitos, dentre outros aspectos.

Sendo assim, a fim de estreitar e diminuir estas dificuldades apontadas, torna-se substancial que desde a graduação os acadêmicos auxiliem os profissionais no campo que estiverem inseridos a prestar uma assistência diferenciada e qualificada através do cuidado humanizado.

\section{CONCLUSÃO}

Conclui-se que os acadêmicos obtiveram experiências exitosas na maternidade e contribuíram para diminuir anseios e dúvidas dos pais. Observou-se que o apoio e suporte da figura paterna junto com a puérpera após o parto, na Golden Hour, proporcionou a formação do vínculo entre os pais e o bebê, uma vez que a paternidade ativa, em todos os momentos da gestação (pré-parto, parto, pós-parto), só traz benefícios positivos para a vida do progenitor, puérpera e bebê.

Sabe-se que a enfermagem tem papel primordial desde o início da gestação até o nascimento no âmbito hospitalar, incentivando e apoiando a participação do progenitor em todas as etapas da gestação. Nos serviços de saúde os profissionais da enfermagem estão em constante evolução quanto ao desenvolvimento de ações educativas para enfatizar a importância do parceiro durante todos os momentos, tornando-o presente em todos as etapas da vida do recém-nascido.

Ademais, a experiência em tela, proporcionou aos acadêmicos de enfermagem um aprimoramento pessoal e principalmente profissional a partir da vivência no setor da maternidade. Dessa forma, os acadêmicos, juntamente aos profissionais do serviço tornaram-se personagens importantes para a quebra de paradigmas do modelo obstétrico vigente, dando visibilidade às boas práticas de atenção ao parto, nascimento e puerpério.

\section{REFERÊNCIAS}

ANTUNES, M. B. et al. Amamentação na primeira hora de vida: conhecimento e prática da equipe multiprofissional. Rev. Av Enferm. v. 35, n. 1, p. 19-29, 2017. 


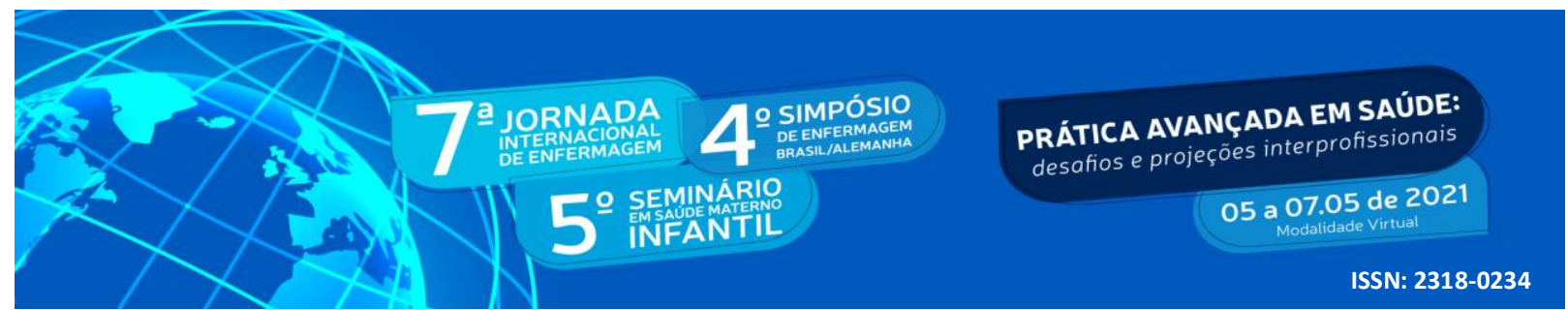

BRASIL, Ministério da Saúde. Mês dos pais destaca participação ativa do homem no prénatal e no cuidado com a criança. Brasília: Ministério da Saúde, 2020.

BRASIL, Ministério da Saúde. PNAISH e paternidade e cuidado. Brasília: Ministério da Saúde, 2016.

BRASIL, Ministério da Saúde. Colocar o bebê para mamar na primeira hora de vida favorece o aleitamento materno. Brasília: Ministério da Saúde, 2014.

BRASIL. Ministério da Saúde. Diretrizes nacionais de assistência ao parto normal. Secretaria de Ciência, Tecnologia e Insumos Estratégicos, Departamento de Gestão e Incorporação de Tecnologias em Saúde. Brasília: Ministério da Saúde, 2017.

BRASIL. Ministério da Saúde. Saúde da criança: crescimento e desenvolvimento. Brasília: Ministério da Saúde, 2012.

CÔRTES, C. T., et al. Implementation of evidence-based practices in normal delivery care. Rev. Latino-Am. Enfermagem. v.26, e2988, 2018.

DONATO, A.D.C., et al. A importância da participação do pai no ciclo gravídico puerperal: uma revisão bibliográfica. Revista Eletrônica da Faculdade de Ceres. v. 4, n. 1, p. 1-15, 2015.

FERREIRA, E. C. Relatos de Experiência. Projeto de Pesquisa de conclusão de Especialização em Atenção ao Paciente Crítico: Urgência, Emergência e UTI (UNINTER). 2019.

OLIVEIRA, A.K. et al. Práticas e crenças populares associadas ao desmame precoce. Rev. Universidad Nacional de Colombia, v.35, n.3, 2017.

OLIVEIRA, C.F. et al. Barreiras à implementação de recomendações para assistência ao parto normal: Revisão rápida de evidências. Rev Panam Salud Publica, v, 44: n.132, 2020.

PINTO, K. R. T. F, et al. Dificuldades na amamentação: sentimentos e percepções paternas. J. Nurs Health., v. 8, n. 1, e. 188106, 2018.

SOUSA, C. M. F. et al. Percepção dos pais sobre sua participação no parto e nascimento. Rev. Enferm. Foco. v. 11, n. 4, p. 29-34, 2020.

WHO. World Health Organization. Recommendations: intrapartum care for a positive childbirth experience. Geneva: WHO, 2018. 